\title{
Influence of patient symptoms and physical findings on general practitioners' treatment of respiratory tract infections: a direct observation study Thomas Fischer*, Susanne Fischer, Michael M Kochen and Eva Hummers- Pradier
}

Address: Department of General Practice, Georg-August-University, Goettingen, Germany

Email: Thomas Fischer* - tfische@gwdg.de; Susanne Fischer - sfische1@web.de; Michael M Kochen - mkochen@gwdg.de; Eva HummersPradier-ehummer@gwdg.de

* Corresponding author

Published: 07 February 2005

BMC Family Practice 2005, 6:6 doi: 10.1 | 86/| 47| |-2296-6-6

This article is available from: http://www.biomedcentral.com/I47/-2296/6/6

(C) 2005 Fischer et al; licensee BioMed Central Ltd.

This is an Open Access article distributed under the terms of the Creative Commons Attribution License (http://creativecommons.org/licenses/by/2.0), which permits unrestricted use, distribution, and reproduction in any medium, provided the original work is properly cited.
Received: 13 August 2004

Accepted: 07 February 2005

\begin{abstract}
Background: The high rate of antibiotic prescriptions general practitioners (GPs) make for respiratory tract infections (RTI) are often explained by non-medical reasons e.g. an effort to meet patient expectations. Additionally, it is known that GPs to some extent believe in the necessity of antibiotic treatment in patients with assumed bacterial infections and therefore attempt to distinguish between viral and bacterial infections by history taking and physical examination. The influence of patient complaints and physical examination findings on GPs' prescribing behaviour was mostly investigated by indirect methods such as questionnaires.
\end{abstract}

Methods: Direct, structured observation during a winter "cough an cold period" in 30 (single handed) general practices. All 273 patients with symptoms of RTI (age above 14, median 37 years, $51 \%$ female) were included.

Results: The most frequent diagnoses were 'uncomplicated upper RTI/common cold' (43\%) followed by 'bronchitis' (26\%). On average, I.8 (95\%-confidence interval (Cl): I.7-2.0) medicines per patient were prescribed (cough-and-cold preparations in $88 \%$ of the patients, antibiotics in $49 \%$ ). Medical predictors of antibiotic prescribing were pathological findings in physical examination such as coated tonsils (odds ratio (OR) 15.4, 95\%-Cl: 3.6-66.2) and unspecific symptoms like fatigue (OR 3.I, 95\%-Cl I.4-6.7), fever (OR 2.2, 95\%-Cl: I.I-4.5) and yellow sputum (OR 2.I, 95\%-Cl: I.I4.I). Analysed predictors explained $70 \%$ of the variance of antibiotic prescribing $\left(R^{2}=0,696\right)$. Efforts to reduce antibiotic prescribing, e.g. recommendations for self-medication, counselling on home remedies or delayed antibiotic prescribing were rare.

Conclusions: Patient complaints and pathological results in physical examination were strong predictors of antibiotic prescribing. Efforts to reduce antibiotic prescribing should account for GPs' beliefs in those (non evidence based) predictors. The method of direct observation was shown to be accepted both by patients and GPs and offered detailed insights into the GP-patient-interaction. 


\section{Background}

Over-prescribing in respiratory tract infections (RTI) has been the topic of numerous studies in general practice. Explanations for the overuse of antibiotics despite weak scientific evidence are multifaceted and vary from efforts to protect patients from "complicated" courses of disease to assumed patient expectations and to inadequate knowledge of physicians [1-4]. Recent investigations demonstrated that patient symptoms and physical findings were also associated with antibiotic prescribing $[5,6]$. A qualitative study using focus groups reported that general practitioners (GPs) tried to distinguish between viral and bacterial infection by history taking and physical examination [7]. This is in line with a questionnaire-based selfregistration study showing a positive correlation between antibiotic prescribing and diagnoses/symptoms assumed to be associated with bacterial infection (e.g. 'sinusitis', 'tonsillitis' or 'yellow-green sputum') [8]. The antibiotic prescription rate in this study, however, was rather low (28\%) and only few predictive items could be detected. Because self-registration studies are methodically limited (varying documentation quality, predetermining questionnaire items and completion of the forms in the sense of "scientific acceptability" [9], we designed a direct observation study to gain detailed insights into the influence of patient symptoms and physical examination on GPs' therapeutic decision. Additionally, we expected to obtain information about the doctor-patient-dialogue (e.g. about recommended self-medication or behaviour advice).

\section{Methods}

We performed a structured, direct observation-based study. This concept is borrowed from social and cultural anthropology developed in the early 1960s [10]. Starting from an unstructured (qualitative) approach, which enabled open, unbiased data collection, a more systematic, structured concept using checklists (quantitative approach) was developed over time, particularly in nursing research [11-13]. We developed a checklist to record information on the interaction between GPs and patients with suspected RTI. Items included were based on history taking and physical examination protocols and the checklist was evaluated and adapted in a pilot study.

Data collection focused on patient complaints, results of physical examinations, further diagnostic procedures and diagnoses. Physical examination findings were coded according to the level of precision obtained (from 'pathological finding' to 'coated tonsils'). The registration of medication included prescriptions as well as recommendations for over-the-counter medicines (OTC) and the dispensing of drug samples (previously brought in by pharmaceutical representatives). Data were mostly acquired through observation during the consultation.
Potentially participating GPs were randomly selected from a GP register and addressed with a form letter. Of 62 GPs addressed, 30 participated this study (16 located in a medium-sized town in Lower-Saxony and 14 in rural areas of North Rhine-Westphalia). To avoid biasing GPs' behaviour no specific hypotheses were shared with the participants.

GPs were visited for one day by S. F. (medical student at time of observation) during a "cough and cold" period in winter; and all consecutive patients (age above 14 years) with symptoms consistent with RTI were included. All patients were informed that a medical student wanted to participate in the consultation and all of them accepted her attendance.

SAS software (Version 8.1) was used to analyse the data [14]. Respiratory tract infections were classified according to the International Classification for Primary Care (ICPC) [15]: 'upper respiratory tract infection (URTI)/ common cold' (R74), 'sinusitis' (R75), 'tonsillitis' (R76), 'laryngitis' (R77) and 'bronchitis' (R78). Since exacerbations of chronic lung diseases are not defined in ICPC, we constructed a dummy variable (including R91, R95 and R96). Multiple diagnoses were accepted and all diagnoses were recorded as stated after the consultation by the GPs. Multiple logistic regression models were used to test for associations between patient characteristics, symptoms, diagnoses and recommended treatment (stepwise backward elimination, $\alpha=0,05)$. Degree of effect is reported as odds ratios (OR) with 95\% confidence intervals (CI). The most frequent patient complaints (cough, sneezing, sore throat, headache, fever, fatigue, hoarseness, myalgia, earache and facial pain) and pathological findings in physical examination as well as age, smoking status and duration of symptoms were included as predictive variables in the multivariate analyses. Drugs were classified using the Anatomic-Therapeutic-Chemical Classification (ATC) codes [16].

\section{Results \\ Doctors and patients}

GPs' median age was 48 years, their median experience in general practice was 12 years and $17 \%$ of the GPs were female. A total of 273 patients (51\% women) were included (representing $21.4 \%$ of all patients visiting their GP at the time of observation). The median number of included patients per GP was 9. No patient refused participation. Patients' median age was 37 years (range 14 to 88).

\section{Diagnoses}

Upper respiratory tract infections/common cold (URTI), bronchitis, tonsillitis and sinusitis were the dominating diagnoses and $88 \%$ of all patients got at least one of these 
Table I: Frequency of diagnoses

\begin{tabular}{lcc}
\hline Diagnoses & No. of patients & (\% of 273 patients) \\
\hline URTI/common cold & & $(42.9)$ \\
Bronchitis & 117 & $(25.6)$ \\
Sinusitis & 70 & $(12.1)$ \\
Tonsillitis & 33 & $(11.0)$ \\
Acute exacerbation chronic lung diseases & 30 & $(8.8)$ \\
Otitis media & 24 & $(6.3)$ \\
Laryngitis & 17 & $(4.4)$ \\
Other & 12 & $(5.9)$ \\
\hline
\end{tabular}

(multiple diagnoses possible, URTI = upper respiratory tract infection)

Table 2: Drug Treatment (according to ATC-code)

\begin{tabular}{|c|c|}
\hline Main groups & Patients (\%) \\
\hline Cough and cold preparations (R05) & $208(76.2)$ \\
\hline Expectorants, excl. combinations with cough suppressants (R05C) & $163(59.7)$ \\
\hline Other cold combination preparations (R05X) & $57(20.9)$ \\
\hline Cough suppressants, excl. combinations with expectorants (R05D) & $4 \mid(15.0)$ \\
\hline Cough suppressants and expectorants, combinations (R05F) & $14(5.1)$ \\
\hline Antibacterials for systemic use $(\mathrm{JOI})$ & $134(49.1)$ \\
\hline Macrolides and lincosamides (JOIF) & $52(19.0)$ \\
\hline Beta-lactam antibacterials, penicillins (JOIC) & $27(9.9)$ \\
\hline Cough suppressants and expectorants, combinations with antibacterials ${ }^{(*)}$ (R05G) & $25(9.2)$ \\
\hline Other Beta-lactam antibacterials (JOID) & $19(7.0)$ \\
\hline Tetracyclines $(\mathrm{J} \mid \mathrm{A})$ & $7(2.9)$ \\
\hline Quinolone antibacterials (J0IM) & $4(1.5)$ \\
\hline Nasal preparations (ROI) & $43(15.8)$ \\
\hline Anti-Asthmatics (R03) & $21(7.7)$ \\
\hline Throat preparations (R02) & $10(3.7)$ \\
\hline Other (Breast unctions (R04), Echinacea-preparations (L03), otologicals (S02)) & $18(6.6)$ \\
\hline
\end{tabular}

$\left({ }^{(*)}\right.$ mostly tetracyclines, ATC $=$ Anatomic, Therapeutic, Chemical Classification)

diagnoses( Table 1). In total 319 diagnoses were made (40 patients were assigned 2 diagnoses and 3 patients 3 diagnoses).

\section{Self-medication and non-medical therapy}

49 patients (18\%) were asked by their GPs about selfmedication and $83 \%$ of them acknowledged the previous use of OTC- drugs (predominantly symptomatic cough and cold drugs, particularly mucolytics). In $40 \%$ of these patients, GPs prescribed an OTC-preparation of the same ATC-classification. The use of household remedies was addressed in $12 \%$ of the patient encounters and threequarter of the patients confirmed that they tried them before the consultation (inhalation in 36\%, gargling in $27 \%$ and drinking of tea in 15\%).

\section{Drug treatment}

On average 1.8 (95\%-CI: 1.68-1.95) drugs were prescribed per patient (Table 2 ). Only $12 \%$ of the patients left the consultation without a drug prescription, $27 \%$ received prescriptions for 3 and more drugs. The most frequently used drugs were acetylcysteine ( 89 prescriptions) and ambroxole (43 prescriptions). Only 8 patients asked for a specific prescription by themselves and 4 of them received the favoured drug. In total, 17 pharmaceutical samples were handed out to 14 patients (mostly cough and cold preparations). The recommendation to buy an OTC-preparation was given to 7 patients (mostly paracetamol).

\section{Predictors of prescriptions}

As shown in table 3 an association between specific diagnoses and antibiotic prescription rates could be demonstrated. Antibiotic prescription rate was highest in patients with the diagnosis 'tonsillitis' (90\%) and relatively low in patients with the diagnosis 'common cold' (18\%). The prescription rate of cough and cold preparations was above $90 \%$ in all diagnoses except 'tonsillitis'. 
Table 3: Prescription rates of antibiotics and cough and cold preparations

\begin{tabular}{lcc}
\hline \multicolumn{1}{c}{ Diagnoses } & \multicolumn{2}{c}{$\begin{array}{c}\text { Prescription rates (in \%) of } \\
\text { Cough and cold preparations } \\
{[\mathbf{9 5 \%} \mathbf{C I}]}\end{array}$} \\
& $\begin{array}{c}\text { Antibiotics [95\% Cl] } \\
\text { Tonsillitis }\end{array}$ & $55.2[37.1-73.3]$ \\
Bronchitis & $89.7[78.6-99.3]$ & $94.3[85.3-95.9]$ \\
Laryngitis & $77.1[67.3-87.0]$ & $91.7[76.0-100.0]$ \\
Sinusitis & $75.0[50.5-99.5]$ & $90.9[81.1-100.0]$ \\
Acute exacerbation chronic lung disease* & $63.6[47.2-80.0]$ & $95.8[87.8-100.0]$ \\
URTI/ common cold & $50.0[30.0-70.0]$ & $90.6[85.3-95.9]$ \\
\hline all patients & $18.0[11.0-24.9]$ & $87.9[84.0-91.7]$ \\
\hline
\end{tabular}

(URTI = upper respiratory tract infection, *see methods)

Table 4: Influence of patient complaints and physical examination results on antibiotic prescription

\begin{tabular}{lcc}
\hline \multicolumn{1}{c}{ Complaints/Physical examination results } & OR & 95\%-Cl \\
\hline Pathologically altered tonsils in mouth and throat inspection & 15.41 & $3.6-66.16$ \\
Pathological otoscopy findings & 8.85 & $1.16-67.58$ \\
Pathological cervical lymph node palpation findings & 6.24 & $1.97-19.71$ \\
Rales in lung auscultation & 4.29 & $2.09-8.83$ \\
Pathological results in paranasal sinus palpation (sinus tenderness) & 3.20 & $1.38-7.42$ \\
Fatigue & 3.09 & $1.42-6.72$ \\
Wheezing in lung auscultation & 2.91 & $1.17-7.23$ \\
Fever & 2.19 & $1.06-4.54$ \\
Yellow sputum & 2.10 & $1.07-4.14$ \\
\hline
\end{tabular}

(multiple logistic regression, $\mathrm{OR}=$ Odds ratio, $\mathrm{Cl}=$ Confidence interval, for included variables see methods)

Multiple logistic regression analyses demonstrated associations between patient complaints, physical examination results and the prescription of antibiotics (Table 4). The calculated model explained $70 \%$ of the variance of antibiotic prescribing $\left(R^{2}=0,695\right)$. Patients' age, smoking status and symptoms such as cough, sore throat, hoarseness, sputum, sneezing, headache and earache had no influence. Also, GPs' characteristics influenced the prescription rate: Although patients' age was uniformly distributed between younger and older GPs, younger GPs ( $<50$ years) prescribed fewer antibiotics than older ones did (37.3 versus $54.4 \%$ of the patients, $\mathrm{p}<0.05)$. Neither the number of treated patients (measured as number of individual patients treated per quarter of a year) nor the GPs' experience in general practice (measured as time of practicing) had influence on antibiotic prescription rates.

Cough and cold preparations were associated with symptoms such as cough (OR 5.09, 95\%-CI: 2.43-10.69), headache (OR 2.43, 95\%-CI: 1.02-5.83), sneezing (OR 2.39, 95\%-CI: $1.2-4.76)$, and abnormal findings in throat examination (OR 2.16, 95\%-CI: 1.11-4.2). The calculated model explained $19 \%$ of the variance of cough and cold preparation prescriptions $\left(\mathrm{R}^{2}=0.186\right)$.

\section{Non-pharmaceutical treatment}

Recommendations for non-pharmaceutical treatment were given to $35 \%$ patients (increased fluid intake in 19\%, inhalation $18 \%$, bed rest $8 \%$, multiple items possible). The advice of increased fluid intake was given to $27 \%$ of patients with the prescription of acetylcysteine (which is recommended in the package insert). One quarter of smokers were advised to stop smoking.

\section{Sickness certification}

A sickness certification was issued to $57 \%$ of the professionally active patients for a median duration of 4 days. $18 \%$ declined the proposal of a sick note.

\section{Patient rescheduling}

$43 \%$ of the patients were asked to return for a "control visit" and $21 \%$ were informed to revisit if they became 
worse. Patients with the diagnoses 'tonsillitis' (in 63\%) and 'bronchitis' (61\%) were more often asked for a "control visit" than patients with the diagnoses 'URTI/ common cold' (23\%).

\section{Discussion}

Our observational study confirmed the overuse of antibiotics and cough and cold preparations despite the lack of scientific evidence. The analysis of predictive indicators for the prescription showed that antibiotic prescribing was associated with specific patient symptoms and physical examination results whereas the prescription of cough and cold preparations was mostly performed indiscriminately. Efforts to reduce antibiotic prescribing, e.g. recommendations for self-medication, counselling on home remedies or delayed antibiotic prescribing were rare.

\section{Consultation rates for $R T I$}

RTI consultation rates of up to one fifth of all patients seen a day may appear high when compared internationally [17]. However, contacting a GP with symptoms of RTI without appointment is a usual and frequent behaviour of German patients, who tend to frequently consult even for minor complaints. Both cultural factors and a fee-for-service oriented healthcare system are likely to influence consultation rates [18]: On average, German patients visit their GP two times often than e.g. people from the Netherlands or France and even 3 times often than in Sweden [17]. Consequently German GPs see many more patients per day than most of their foreign colleagues. As to our study, one-day visits proved to be sufficient to acquire a representative number of patients.

\section{Antibiotic prescription}

In our study, the demonstrated antibiotic prescription rate was high in comparison to other European investigations and rather approaches US-American levels [2,8]. Parallel to this observation study, a documentation study was performed in Germany using questionnaires to record GPs' behaviour with patients also displaying symptoms of RTI [8]. The results of these two studies differ although the patient characteristics and diagnoses were comparable. One explanation for the lower prescription rates in the documentation study could be a selection bias because all of the GPs in our observation study were randomly recruited, whereas all of the GPs participating in the documentation study were recruited at an EBM-workshop. In addition, the completion of the documentation sheets in the sense of "scientific acceptability" is a known confounder $[8,9]$. However, it can be argued that the direct observation method also influenced the prescription rate ("observer effect" or "Hawthorne effect") [19]. It appears likely that the intense public discussion in the last years about the overuse of antibiotics in primary care would rather lower the rates.
In our observation, patient complaints and physical findings such as 'pathologically altered tonsils' or 'wheezing/ rales' in lung auscultation have been shown to be strong predictors of GPs prescribing behaviour comparable to recent investigations [5,6]. Besides clear evidence-based medical facts, more unspecific factors such as fever or fatigue caused GPs to prescribe antibiotics, as well. Furthermore, 'yellow sputum' was positively associated with antibiotic prescription as demonstrated in other studies before [8], although this symptom has shown to be unspecific in predicting bacterial infections (in acutely ill patients) $[20,21]$. It appears that GPs' behaviour is influenced by empirical "internal evidence" (to detect bacterial infections), which - at least partially -, contradicts actual scientific "external evidence" $[4,5,22]$. The extensive impact of those predictors (explaining $70 \%$ of the variance of antibiotic prescribing) demonstrates that efforts to reduce high antibiotic prescribing rates in RTI must focus on GPs' beliefs in pathogenesis and the misattributing of clinical signs and patient symptoms to bacterial infection.

Diagnoses made by GPs were strong predictors of prescribing behaviour. Patients with the diagnosis 'URTI/ common cold' received significantly fewer antibiotics than those with 'tonsillitis', 'sinusitis' or 'bronchitis'. Despite the controversial scientific evidence for antibiotic therapy in those conditions, the majority of participating GPs seemed to be convinced that antibiotics were necessary or likely to be helpful $[4,5,22]$. Furthermore, patients with these diagnoses were frequently rescheduled for a "control visit" probably reflecting GPs' beliefs about "potentially dangerous diseases".

Complementary to quantitative analysis direct observation offered interesting insights in the GP-patient-consultation. Only one patient asked for an antibiotic prescription, which is in contrast to the common belief about patient expectations [3,23]. Surprisingly, the observer noticed only few efforts of GPs to reduce antibiotic prescription rates. The questionable benefit of antibiotics was discussed in very few consultations only and no doctor used delayed prescribing which has been demonstrated to be a useful tool to reduce antibiotic intake [24].

The impact of non-medical reasons of prescribing should not be disregarded [25]. Our observational study survey could not measure the impact of tacit or implicit beliefs and relational aspects on the very complex issue of motivation for prescribing. However, the importance of nonmedical reasons (e.g. patients' expectations or GPs perception of those expectations) as an independent predictor of antibiotic prescribing has already been shown in several studies $[3,5,7,23,26]$. Additional influences of the healthcare system seem probable: fee-for-service remuneration, liberal access to GPs and specialist may result in GPs fear- 
ing to lose patients (and money) when denying a prescription.

\section{Prescription of cough and cold preparations}

Prescription of cough and cold preparations in patients with acute RTI is not supported by scientific evidence [2729], in particular for the expectorants acetylcysteine and ambroxole, which are the most commonly used cough and cold preparations in Germany. Although some investigations have demonstrated an effect of those substances on chronic bronchitis, evidence in acute RTI is so far absent because well-performed control trials of ambroxole and acetylcysteine are rare [30].

International comparisons of OTC-prescription rates are restricted because of different health insurance systems (and refunding policies) in different countries. Recent data from Belgium - a country with an at least partially comparable health system - showed high prescription rates for cough and cold preparations, too [31]. Interestingly, Belgium GPs preferred prescribing of cough suppressants rather than expectorants, which is the leading group in Germany (Table 2).

The fact that only $19 \%$ of the variance of cough and cold preparation prescriptions was explained by clinical signs and patient symptoms supports the assumption that nonmedical reasons dominated the decision to prescribe. Frequently, the term "pseudo-placebo" is used in this context to describe the function of those preparations [32] and assumed patient expectations were mainly held responsible for the prescription $[3,23]$.

Eighty-three percent of patients asked confirmed the use of OTC-preparations before the consultation, which were often identical with the subsequently prescribed preparations. GPs did not appreciate the opportunity to encourage this self-medication and cost reducing patient behaviour, perhaps because they were afraid that patients would not consult them with similar complaints at another time [33]. In this context, it must be noted that, in contrast to the UK, German GPs are paid only for patients actually consulting them.

\section{Conclusions}

The method of direct observation allowed for detailed and sensitive insights in GP-patient-consultations. Nevertheless, investigators must be aware of the specific disadvantages of the method (e.g. the "observer effect").

Furthermore, we could demonstrate that patient complaints and physical examination results had a strong impact on GPs prescribing behaviour, especially on antibiotic prescription. Although this GP behaviour is not in accordance with actual scientific evidence, GPs' under- standing of pathogenesis and the value of clinical signs should be strongly considered when efforts are made to reduce the overuse of antibiotics in primary care.

\section{List of abbreviations}

ATC code = "Anatomic, Therapeutic, Chemical Classification" code

$$
\begin{aligned}
& \mathrm{CI}=\text { Confidence interval } \\
& \text { GP }=\text { General practitioner } \\
& \text { OR = Odds ratio } \\
& \text { RTI = Respiratory tract infection } \\
& \text { URTI = Upper respiratory tract infection }
\end{aligned}
$$

\section{Competing interests}

The author(s) declare that they have no competing interests.

\section{Authors' contributions}

TF participated in the study design, analysis, interpretation of data and drafted the manuscript. SF participated in the study design, analysis, interpretation of data, draft of manuscript and performed the practice observations. MMK participated in the study design and draft of manuscript. EHP participated in the study design, analysis, interpretation of data and draft of the manuscript. All authors read and approved the final manuscript.

\section{References}

I. Mainous AG, Hueston WJ, Clark JR: Antibiotics and upper respiratory infection: do some folks think there is a cure for common cold? J Fam Pract 1996, 42:357-36I.

2. Gonzales R, Steiner JF, Sande MA: Antibiotic prescribing for adults with colds, upper respiratory tract infections, and bronchitis by ambulatory care physicians. JAMA I997, 278:901-904.

3. Himmel W, Lippert-Urbanke E, Kochen MM: Are patients more satisfied when they receive a prescription? The effect of patient expectations in general practice. Scand J Prim Health Care 1997, I5: I 18-122.

4. Butler CC, Rollnick S, Pill R, Maggs-Rapport F, Stott N: Understanding the culture of prescribing: qualitative study of general practitioners' and patients' perceptions of antibiotics for sore throats. BMJ 1998, 3 I 7:637-642.

5. Dosh SA, Hickner JM, Mainous AG, Ebell MH: Predictors of antibiotic prescribing for nonspecific upper respiratory infections, acute bronchitis, and sinusitis. J Fam Pract 2000, 49:407-4I4.

6. Fagnan LJ: Prescribing antibiotics for upper respiratory infections. J Fam Pract 2000, 5:4I5-4I7.

7. Coenen S, van Royen P, Vermeire E, Hermann I, Denekens J: Antibiotics for cough in general practice: a qualitative decisions analysis. Fam Pract 2000, 17:380-385.

8. Hummers-Pradier E, Pelz J, Himmel W, et al.: Treatment of respiratory tract infection - a study in 18 general practices in Germany. Eur J Gen Pract 1999, I: 15-20.

9. Rethans JJ, Westin S, Hays R: Methods for quality assessment in general practice. Fam Pract 1996, I3:468-476.

10. Gold RL: Roles in sociological field observations. Soc Forces 1958, 36:217-223. 
II. Pretzlik U: Observational methods and strategies. Nurs Res 1994, 2:13-2|.

12. Turnock $C$, Gibson $V$ : Validity in action research: a discussion on theoretical and practice issues encountered whilst using observation to collect data. J Adv Nurs 200I, 36:47I-477.

13. Mulhall $A$ : In the field: notes on observation in qualitative research. J Adv Nurs 2003, 4I:306-3I3.

14. SAS Institute Inc: SAS/STAT. User's Guide Version 8. Cary, NC 1999.

15. Lamberts $\mathrm{H}$, Wood M, Hofmans-Okkes, ed: The international classification of primary care in the European community. Oxford University Press, Oxford-New York-Toronto; 1993.

16. Schwabe U: ATC-Code: anatomic-therapeutic chemical classification for the German drug market (in German). Bonn: GKV-Arzneimittelindex, WIdO 1995.

17. German Federal Ministry of Health and Social Security [http://www.bmgs.bund.de/deu/gra/aktuelles/pm/bmgs04/ $51505331 . \mathrm{cfmm}]$. (in German)

18. Turkie P: "French lessons". BMJ 2004, 329:1393.

19. Mangione-Smith R, Elliott MN, McDonald L, McGlynn EA: An observational study of antibiotic prescribing behaviour and the Hawthorne effect. Health Serv Res 2002, 37:1603-1623.

20. Winther B, Brofeldt S, Gronborg H, et al.: Study of bacteria in the nasal cavity and nasopharynx during naturally acquired common colds. Acta Otolaryngol 1984, 98:31 5-320.

21. Gonzales R, Barret PH, Steiner JF: The relation between purulent manifestations and antibiotic treatment of upper respiratory tract infections. J Gen Intern Med 1999, 14:151-156.

22. Vinson D, Lutz I: The effect of parental expectation on treatment of children with cough. J Fam Pract 1993, 37:23-27.

23. Cockburn J, Pit S: Prescribing behaviour in clinical practice: patients' expectations and doctors' perceptions of patients' expectations - a questionnaire study. BMJ 1997, 3 I 5:520-523.

24. Dowell J, Pitkethly M, Bain J, Martin S: A randomised controlled trial of delayed antibiotic prescribing as a strategy for managing uncomplicated respiratory tract infection in primary care. Br J Gen Pract 200 I, 5 I :200-205.

25. Butler CC, Rollnick S, Kinnersley P, Jones A, Stott N: Reducing antibiotics for respiratory tract symptoms in primary care: consolidating 'why' and considering 'how'. Br J Gen Pract 1998, 48: $1865-1870$.

26. Coenen S, Michiels B, Van Royen P, Van der Auwera JC, Denekens J: Antibiotics for coughing in general practice: a questionnaire study to quantify and condense the reasons for prescribing. BMC Family Practice 2002, 3:16.

27. Del Mar C, Glasziou P: Upper respiratory tract infection. Clin Evidence 2003, 9: I70I-I7II.

28. Poole PJ, Black PN: Mucolytic agents for chronic bronchitis or chronic obstructive pulmonary disease. The Cochrane Database of Systematic Reviews 2003, I : . Art. No. CD00I 287

29. Schroeder K, Fahey T: Over-the-counter medications for acute cough in children and adults in ambulatory settings. The Cochrane Database of Systematic Reviews 2004, 4:. Art. No. CD001831.pub2

30. Matthys H, de Mey C, Carls C, Rys A, Geib A, Wittig T: Efficacy and tolerability of Myrtol standardized in acute bronchitis. A multi-centre, randomised, double-bilnd, placebo-controlled parallel group clinical trials vs. cefuroxime and ambroxole. Arzneimittelforschung 1999, 50:700-II.

31. Coenen S, Van Royen P, Michiels B, Denekens J: Optimizing antibiotic prescribing for acute cough in general practice: a cluster-randomized controlled trial. J Antimicrobial Chemotherapy 2004, 54:66I-672.

32. Kochen MM: General practice characteristics of pharmacotherapy (in German). Z Arztl Fortbild 1994, 88:647-654.

33. Himmel W: Self-medication - economic, sociopharmacologic and toxicologic aspects (in German). Dtsch Med Wschr 2000, | 25:40|-407.

\section{Pre-publication history}

The pre-publication history for this paper can be accessed here:

http://www.biomedcentral.com/1471-2296/6/6/prepub

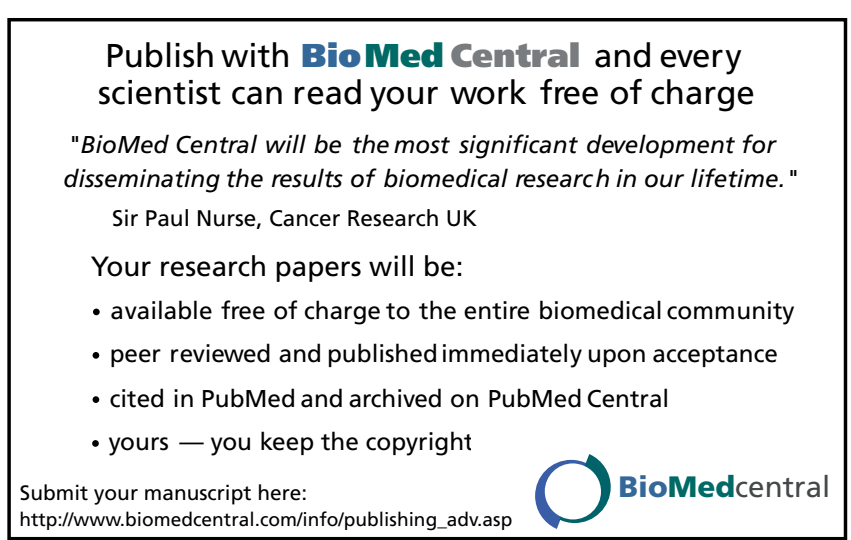

